\title{
Whole-Microwave System Modeling for Brain Imaging
}

\author{
Ibtissam El Kanfoud ${ }^{1}$, Victorita Dolean ${ }^{1,4}$, Claire Migliaccio ${ }^{1}$, Jerôme Lanteri ${ }^{1}$, Iannis Aliferis ${ }^{1}$, Christian Pichot ${ }^{1}$, \\ Pierre-Henri Tournier ${ }^{2}$, Frédéric Nataf ${ }^{2}$, Frédéric Hecht ${ }^{2}$, Serguei Semenov ${ }^{3}$, Marcella Bonazzoli ${ }^{4}$, Francesca Rapetti ${ }^{4}$, \\ Richard Pasquetti ${ }^{4}$, Maya de Buhan ${ }^{5}$, Marie Kray ${ }^{6}$, Marion Darbas \\ ${ }^{1}$ Univ. Nice Sophia Antipolis, CNRS, LEAT, UMR 7248, 06900 Sophia Antipolis, France \\ ${ }^{2}$ INRIA, Alpines, UPMC, LJLL, UMR 7598, 75000, Paris, France \\ ${ }^{3}$ EMTensor GmbH, TechGate, 1220 Vienna, Austria \\ ${ }^{4}$ Univ. Nice Sophia Antipolis, CNRS, LJAD, UMR 7351, 06000 Nice, France \\ ${ }^{5}$ Univ. Paris Descartes, MAP5, UMR 8145, 75000 Paris, France \\ ${ }^{6}$ Mathematishes Institut, Universität Basel, Switzerland \\ ${ }^{7}$ Univ. Picardie Jules Verne, LAMFA, UMR CNRS 7352, Amiens, France \\ ibtissam.el-kanfoud@unice.fr
}

\begin{abstract}
In this paper, we present the results of a wholesystem modeling of a microwave measurement prototype for brain imaging, consisting of 160 ceramic-loaded antennas working around $1 \mathrm{GHz}$. The modelization has been performed using open source FreeFem++ solver. Quantitative comparisons were performed using commercial software Ansys-HFSS and measurements. Coupling effects between antennas are studied with the empty system (without phantom) and simulations have been carried out with a fine numerical brain phantom model issued from scanner and MRI data for determining the sensitivity of the system in realistic configurations.
\end{abstract}

Keywords: Microwave Tomography; FreeFem++; Stroke Detection; Brain Imaging.

\section{INTRODUCTION}

Microwave tomography is a novel, early development stage imaging modality with a large number of potential attractive medical applications. A significant difference between properties of normal and diseased tissue is a great potential of this imaging modality. By exposing head tissues to low-level microwave incident field and capturing the scattered field by an array of antennas, the estimation of the dielectric properties of the brain tissues can be approximated and a diagnosis inferred. Although there are numerous medical conditions that could be compatible with a microwave imaging system, the stroke is perhaps of most significance. A stroke is disturbance in the blood supply to the brain caused by either a blocked or burst blood vessel. A stroke can be cast in two major categories, ischemic and hemorrhagic. A majority of these works have made use of geometrical simple phantoms with only a limited amount of tissue-mimicking materials. Therefore, there are still doubts on the feasibility of stroke imaging as the aforementioned works on stroke imaging and detection have yet applied on a more realistic phantom. To that end, this work will examine this application. This needs an accurate modeling of the interaction of an incident electromagnetic wave with a realistic model of head.

The main goal of this study is the methodological and numerical development of a new robust inversion tool (associated with the electromagnetic forward problem). This implies the development of a general parallel open source simulation code of a direct problem, based on the high-level integrated development environment of FreeFem++, which can be used for modeling the scattering of arbitrary electromagnetic waves in highly heterogeneous media, over the frequency range of the measurement system.

Developing a numerical tool for solving the forward problem to realize a realistic model of the data acquired by the microwave imaging system is novel and challenging [1]. The modeling must have to take account accurately of the high heterogeneity and complexity of head tissues (skin, fat, skull, bone marrow, brain/white matter, brain/grey matter, cerebrospinal fluid, arteries, etc.) for normal cases and for different possible brain pathology cases (ischemic and hemorrhagic strokes, brain injuries, etc.). The wave/matter interaction must take also in account accurately of the incident field from the transmitting antennas. This interaction is very complex, as it must be seen as a coupling problem between the antennas and the head rather than a simple scattering problem. In other words, the presence of the head disrupts the incident field created by the antennas. In addition, the electric field is measured by means of sensors (antennas). Therefore, we don't have access directly to the electric field but through antennas. Looking at the state-of-the-art of numerical modeling, the whole interaction has never been modeled. Only simple models (using plane waves, with simple antenna models and without taking into account the coupling) have been carried out in the literature.

\section{MODELING}

\section{A. Modeling using FreeFEM ${ }^{++}$}

FreeFem++ (http://www.freefem.org/ff ++ ) ) is a partial differential equation solver that has its own language. 
FreeFem++ has an advanced automatic mesh generator, capable of a posteriori mesh adaption; it uses fast algorithms such as the multi-frontal method UMFPACK, Super LU. Hyperbolic and parabolic problems are solved using iterative algorithms prescribed by the user, with FreeFem ++ high-level language. Several triangular finite elements, including discontinuous elements are available. A key and, to our knowledge, unique feature of FreeFem++ is the possibility to interpolate between two arbitrary finite element spaces even if defined on different meshes. Many finite elements are already implemented, among them Nedelec finite elements and Discontinuous Galerkin (DG) discretization.

We will compare the results obtained using FreeFem++ with Ansys-HFSS in order

- To determine HFSS limitations for modeling the whole empty system as commercial tools are getting increasingly powerful but limited when the computation volume is too large.

- Furthermore, different simulations will be performed using accurate numerical brain phantom that may increase the complexity and computation volume.

\section{B. Modeling the whole measurement system with HFSS (using direct solver)}

The Transmitter-receiver (T/R) system [1] is composed of 160 antennas: 5 rings of 32 open ceramic-loaded rectangular waveguides around a cylindrical metallic tank (Fig. 1). The horizontal spacing between each ring is $30 \mathrm{~mm}$. Each antenna can act as Transmitter/Receiver. When an antenna is transmitting, a wave port representing the fundamental $\mathrm{TE}_{10}$ mode excitation is used.

The height and diameter of the metallic cylindrical cylinder are, respectively, $280 \mathrm{~mm}$ and $285 \mathrm{~mm}$. The bottom of the metallic cylinder is positioned at a distance of $100 \mathrm{~mm}$ from the 5th ring. The first ring is placed at $30 \mathrm{~mm}$ from the top of the cylinder [1].

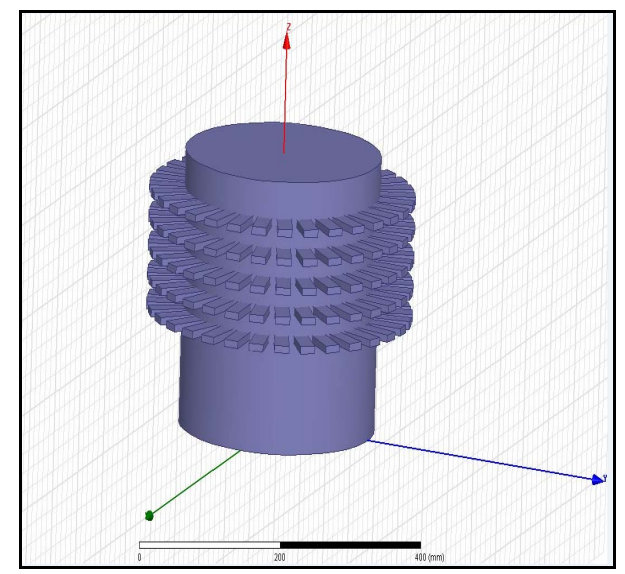

Fig 1. Transmitter/Receiver system with 5 rings of 32 ceramic-loaded rectangular waveguides
The cylinder is filled with a matching medium. For example, we have used a liquid having the relative complex permittivity value of $\varepsilon_{\mathrm{r}}=44-\mathrm{i} 20$ measured at $1 \mathrm{GHz}$. The waveguide dimensions are $21 \times 7.5 \mathrm{~mm}^{2}$ and the dielectric properties of ceramic are assumed to be lossless with $\varepsilon_{\mathrm{r}}=59$ [1].

For the radiation boundary, the distance from the whole system is $\lambda / 4$ in all directions.

Hereafter, are shown the electric field distribution corresponding to different meshes when one antenna of ring no2 is transmitting and all antennas are receiving. Nevertheless, only two rings at a time were modeled due to the large computation memory required. Fig. 2 shows the field distribution when an antenna of ring no. 2 is transmitting in presence of ring no.1 (Fig. 2a) with associated mesh grid (Fig. 2b).

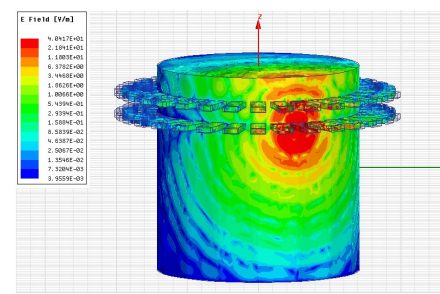

Fig. 2a

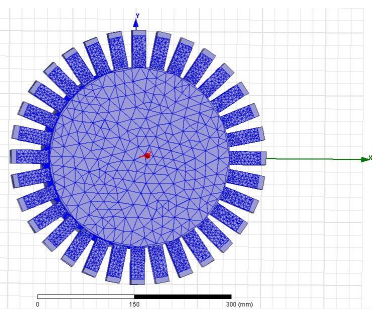

Fig. 2b

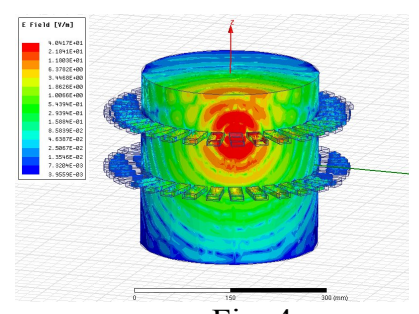

Fig. 4a

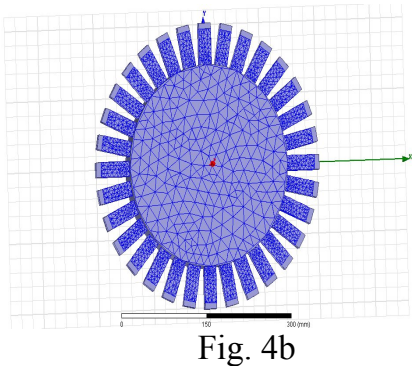

Fig. 4b

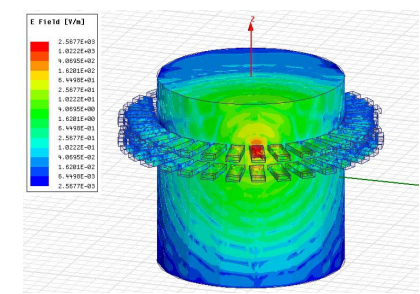

Fig. 3a

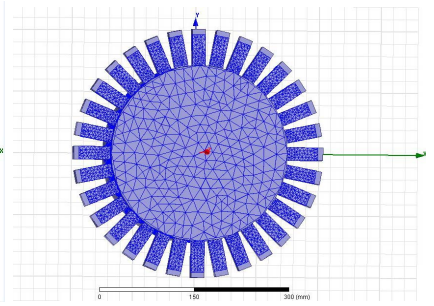

Fig. 3b

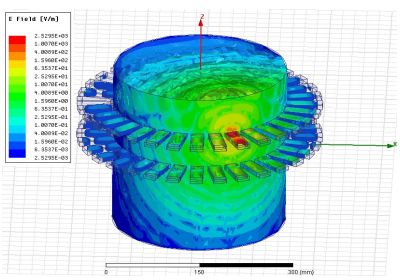

Fig. $5 \mathrm{a}$

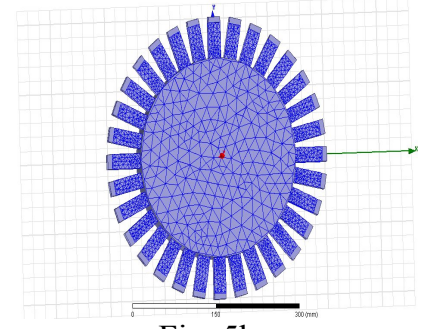

Fig. 5b 


\begin{tabular}{|l|l|}
\hline $\begin{array}{l}\text { Mesh corresponding to Fig. } \\
2 \mathrm{~b} \\
\text {-Total number of mesh } \\
\text { elements: } 94493\end{array}$ & $\begin{array}{l}\text { Mesh corresponding to Fig. } \\
\text {-Total number of mesh } \\
\text { elements: } 159910\end{array}$ \\
\hline $\begin{array}{l}\text { Mesh corresponding to } \\
\begin{array}{l}\text { Fig. } 4 \mathrm{~b} \\
\text {-Total number of mesh } \\
\text { elements is } 164224\end{array}\end{array}$ & $\begin{array}{l}\text { Mesh corresponding to } \\
\text { Fig. } 5 \mathrm{~b}\end{array}$ \\
\hline
\end{tabular}

Even if HFSS generates an initial mesh, the numbers of final mesh grid elements indicated above are the final mesh elements after automatic refining.

\section{Modeling the whole measurement system with FreeFem++ (using domain decomposition methods)}

The open source software FreeFem++ is a Domain-specific language (DSL) base on C++. It is mainly used for solving PDE with Galerkin method. The advantage of using a DSL rather than a low level language is not only in its simplicity to use when it comes to resolve tridimensional problems but as well as for its good speed of execution in comparison to lowlevel languages. Over the versions, FreeFem++ has been linked to numeric linear algebra libraries for the resolution of linear systems and also for the computation of eigenvalues. The parallelism is done by the The MPI programming provides parallelism on supercomputing machines [2]. The method used is based on Schwarz domain decomposition method. The principle consists of dividing large size problems into sub-problems of lower size, and thus simpler to solve.

The first step consists of setting up the software Free FEM++ for processing the complete system. Then the second step was to solve Maxwell equations in FreeFem++. The third step was to simulate the measured $\mathrm{S}_{\mathrm{ij}}$ coefficients by the experimental system using the Hermitian scalar product between the electric field calculated by FreeFem++ at the ith waveguide interface and the electric field of the fundamental mode $\mathrm{TE}_{10}$ of the waveguide. The fourth step was to simulate the whole-system with a virtual numerical head issued from scanner and MRI images with $0.5 \mathrm{~mm}$ spatial resolution. Finally, we compare the results between HFSS, FreeFem++ and the measurements. As for HFSS, we use the open software FreeFem++ for modeling the whole measurement system composed with 160 waveguides when one antenna of ring no2 is transmitting and all antennas are receiving.

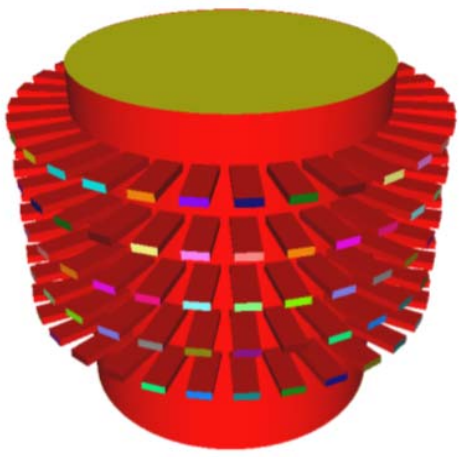

Fig 6. Transmitter/Receiver system with 5 rings of 32 ceramic-loaded rectangular waveguides with FreeFem++

\section{Formulation}

For a single frequency excitation, the propagation and scattering of electromagnetic waves inside the whole imaging chamber tank is described by the second order time-harmonic Maxwell's equations.

$$
-k^{2}+\nabla \times(\nabla \times E)=0 \text { with } k=2 \pi f \sqrt{\varepsilon_{0} \varepsilon_{r} \mu_{0}}
$$

Where $\varepsilon_{0}$ and $\mu_{0}$ are, respectively, the permittivity and permeability of free space permittivity, and $\varepsilon_{\mathrm{r}}$ the relative permittivity.

\section{E. Boundary conditions}

\section{1) Metallic boundary conditions}

Dirichlet boundary condition represented by equation

$$
E \times n=0
$$

2) Absorbing Boundary Condition

Impedance boundary condition for receivers

$$
\nabla \times E \times n-i k n \times(E \times n)=0
$$

3) Impedance boundary condition

Impedance boundary condition for transmitter excitation

$$
\nabla \times E \times n-i k n \times(E \times n)=g^{i n c}
$$

\section{F. System with phantom and system without phantom}

1) Simulation of the brain modal inside the measurement system.

- $f=1 \mathrm{GHz}, \mathrm{Gel}: \mathcal{E}_{r}=44-i 20$

Virtual numerical head issued from scanner and MRI images with $0.5 \mathrm{~mm}$ spatial resolution.

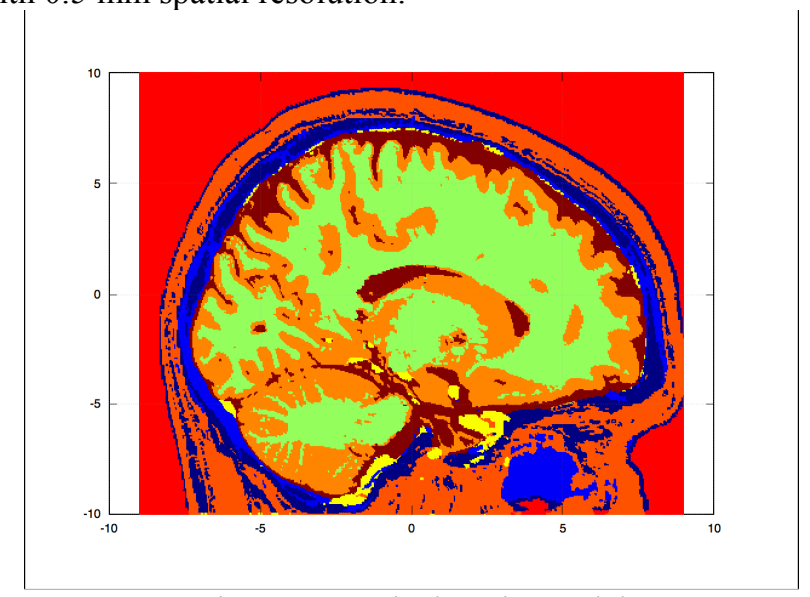

Fig. 7. Numerical Brain Model

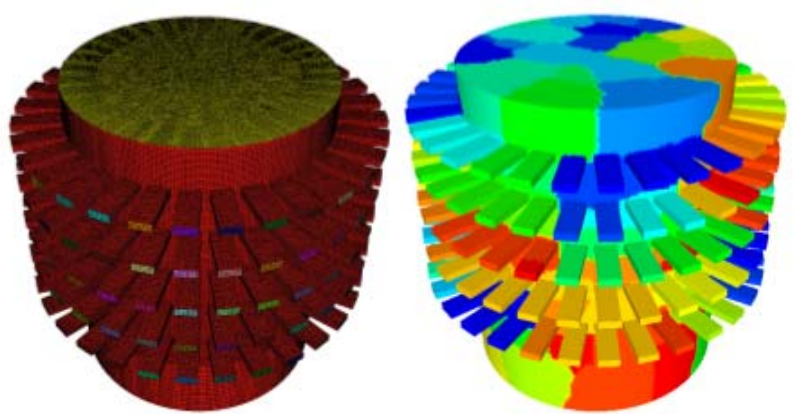

Fig. 8. Mesh of the domain (left), divided into 128 subdomains by METIS (right) 

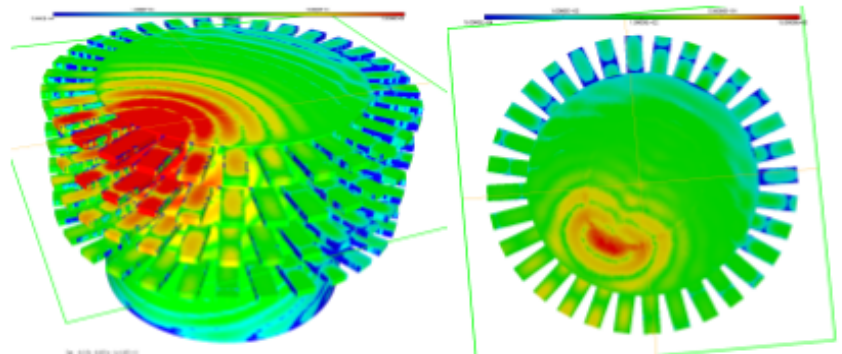

Fig. 9. Electric field with gel only (left), field scattered by the head (right)

\section{RESULTS}

We present the amplitude and phase results, over observation angle using open source FreeFem++ solver. Quantitative comparisons are performed using commercial software AnsysHFSS and measurements.

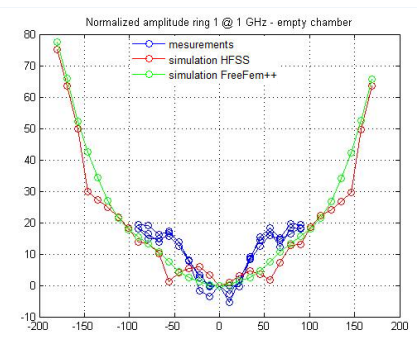

Fig. 10 Amplitude of ring no.1

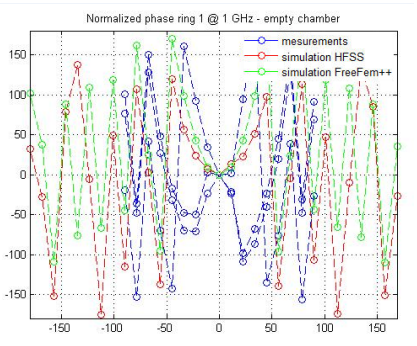

Fig. 11 Phase of ring no. 1
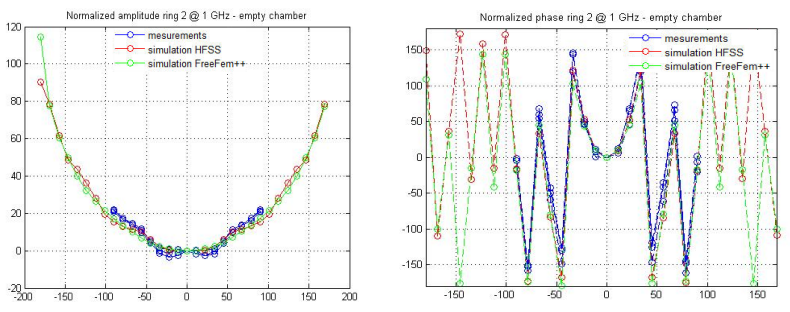

Fig .12. Amplitude of ring no.2 Fig.13. Phase of ring no.2
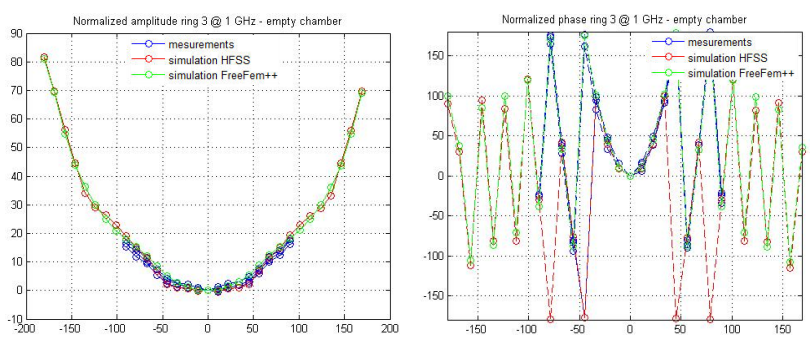

Fig.14. Amplitude of ring no.3
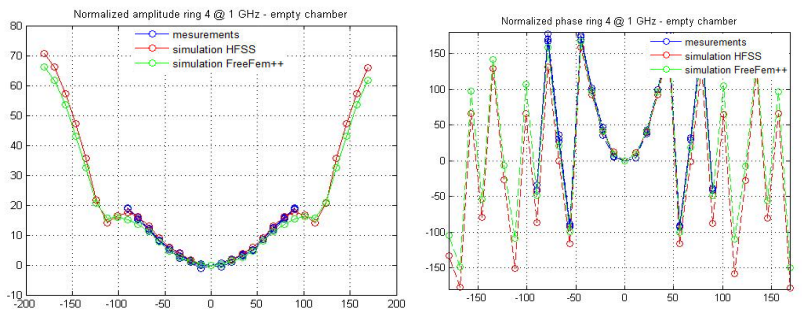

Fig.16. Amplitude of ring no.4

Fig. 17. Phase of ring no.4

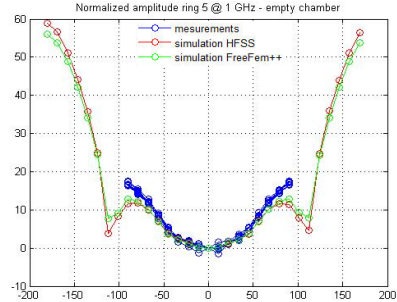

Fig.18. Amplitude of ring no.5

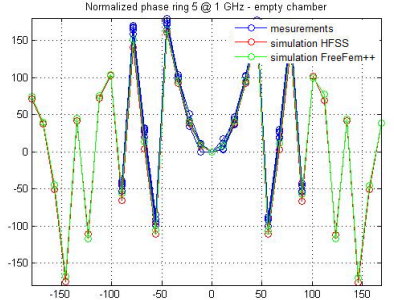

Fig. 19. Phase of ring no.5
The results show an excellent agreement for rings 2, 3, 4 and 5 between HFSS, FreeFem++ and measurements. There is a slight discrepancy for the first ring between measurements and the numerical results for both HFSS and Freefrem++.

\section{CONCLUSION}

The modeling of the whole-microwave imaging system has been performed using open source software FreeFem++. The results have been compared with Ansys-HFSS and measurements. They show a very good agreement.

\section{ACKNOWLDGEMENT}

This work was supported by ANR project MEDIMAX (ANR13-MONU-0012).

\section{REFERENCES}

[1] S. Semenov, B. Seiser, E. Stoegmann, and E. Auff, "Electromagnetic tomography for brain imaging: from virtual to human brain", 2014 IEEE Conf. Antenna Measur. \& Appl. (CAMA), pp. 1-4, Nov. 2014.

[2] F. Hecht, "New development in FreeFem++", J. Numer. Math. 20, no. 3-4, pp. 251-265, (2012). 